\title{
TRPM7 Is Required within Zebrafish Sensory Neurons for the Activation of Touch-Evoked Escape Behaviors
}

\author{
Sean E. Low, ${ }^{1,2}$ Kimberly Amburgey, ${ }^{1}$ Eric Horstick, ${ }^{1}$ Jeremy Linsley, ${ }^{1}$ Shawn M. Sprague, ${ }^{1}$ Wilson W. Cui, ${ }^{1}$ \\ Weibin Zhou, ${ }^{1}$ Hiromi Hirata, ${ }^{1}$ Louis Saint-Amant, ${ }^{1,2}$ Richard I. Hume, ${ }^{1}$ and John Y. Kuwada ${ }^{1}$ \\ ${ }^{1}$ Department of Molecular, Cellular and Developmental Biology, University of Michigan, Ann Arbor, Michigan, 48109-1048, and ²Départment de Pathologie \\ et Biologie Cellulaire, Université de Montréal, Montréal, Quebec, Canada
}

Mutations in the gene encoding TRPM7 (trpm7), a member of the Transient Receptor Potential (TRP) superfamily of cation channels that possesses an enzymatically active kinase at its $\mathrm{C}$ terminus, cause the touch-unresponsive zebrafish mutant touchdown. We identified and characterized a new allele of touchdown, as well as two previously reported alleles, and found that all three alleles harbor mutations that abolish channel activity. Through the selective restoration of TRPM7 expression in sensory neurons, we found that TRPM7's kinase activity and selectivity for divalent cations over monovalent cations were dispensable for touch-evoked activation of escape behaviors in zebrafish.

Additional characterization revealed that sensory neurons were present and capable of responding to tactile stimuli in touchdown mutants, indicating that TRPM7 is not required for sensory neuron survival or mechanosensation. Finally, exposure to elevated concentrations of divalent cations was found to restore touch-evoked behaviors in touchdown mutants. Collectively, these findings are consistent with a role for zebrafish TRPM7 within sensory neurons in the modulation of neurotransmitter release at central synapses, similar to that proposed for mammalian TRPM7 at peripheral synapses.

\section{Introduction}

TRPM7, a member of the Transient Receptor Potential (TRP) superfamily of cation channels, represents one of only two known ion channels to possess an enzymatically active kinase tethered to its C terminus (Montell, 2005). Since the identification of this unusual pairing of an ion channel with a kinase (Runnels et al., 2001), several studies have been undertaken to determine whether TRPM7's kinase regulates channel activity. From these studies (Nadler et al., 2001; Schmitz et al., 2003; Matsushita et al., 2005), TRPM7's kinase activity has been deemed dispensable for channel function. However, these studies have been performed in heterologous expression systems, and therefore the contribution

Received Sept. 21, 2010; revised June 8, 2011; accepted June 15, 2011.

Author contributions: S.E.L., R.I.H., and J.Y.K. designed research; S.E.L., K.A., E.H., J.L., and R.I.H. performed research; S.M.S., W.W.C., W.Z., H.H., and L.S.-A. contributed unpublished reagents/analytic tools; S.E.L., K.A., and R.I.H. analyzed data; S.E.L., R.I.H., and J.Y.K. wrote the paper.

This work was supported by the National Institute of Neurological Disorders and Stroke (Grant R01NS54731 to J.Y.K.), the National Science Foundation (Grant 0725976 to J.Y.K.), a Long-Term Fellowship from the Human Frontier Science Program (to H.H.), and in part by the Center for Organogenesis Training Grant 5-T32-HD007505 (to W.W.C.). We thank Haoxing $\mathrm{Xu}$ and members of the Kuwada and Hume laboratories for helpful comments regarding the preparation of this manuscript. We also thank Dr. Hitoshi Okamoto (RIKEN, Wako-city Saitama, Japan) for the zCREST2 enhancer element and the ssx-mini-ICP:eGFP stable transgenic line of zebrafish, Dr. Paul Henion (The Ohio State University, Columbus, $\mathrm{OH})$ for touchdown allele $b 508\left(t d 0^{b 508}\right)$, and The Zebrafish International Resource Center (P40 RR12546 from NIH-NCRR) for touchdown allele $t d 0^{\text {tz310c }}$

S. E. Low's present address: The Rockefeller University, New York, NY.

W. W. Cui's present address: University of California, San Francisco School of Medicine, San Francisco, CA.

W. Zhou's present address: Life Science Institute, University of Michigan, Ann Arbor, MI.

H. Hirata's present address: Center for Frontier Research, National Institute of Genetics, Mishima, Japan.

Correspondence should be addressed to John Y. Kuwada, University of Michigan, Kraus Natural Science Building, 830 N. University Avenue, Ann Arbor, MI 48109-1048. E-mail: kuwada@umich.edu.

DOI:10.1523/JNEUROSCI.4950-10.2011

Copyright $\odot 2011$ the authors $\quad 0270-6474 / 11 / 3111633-12 \$ 15.00 / 0$ of kinase activity to channel function in a native cell in vivo has yet to be addressed.

Beyond a potential interplay between kinase activity and channel function, other studies have sought to elucidate the cellular role(s) for this dual function protein that is conserved among vertebrates. These studies in native cells using pharmacological agents and knock-out strategies have, however, been hampered by the absence of TRPM7-specific antagonists and the finding that the loss of trpm 7 in mice is embryonically lethal (Jin et al., 2008). Despite these hurdles, TRPM7 kinase activity has been linked to the phosphorylation of several intracellular proteins (Dorovkov and Ryazanov, 2004; Clark et al., 2006, 2008), while TRPM7 channel activity has been implicated in a wide range of cellular processes including mechanotransduction $(\mathrm{Nu}-$ mata et al., 2007a,b), transmitter release (Krapivinsky et al., 2006; Brauchi et al., 2008), pacemaker activity of gut cells (Kim et al., 2005), and cell survival (Nadler et al., 2001) via $\mathrm{Mg}^{2+}$ homeostasis (Schmitz et al., 2003). However, more recent results have cast doubt as to TRPM7's role in $\mathrm{Mg}^{2+}$ homeostasis (Jin et al., 2008). Thus, questions regarding the function of TRPM7 are still outstanding.

Our investigation of TRPM7 began with the isolation of a touch-unresponsive zebrafish mutant that was subsequently mapped to the touchdown locus, the gene known to encode TRPM7 (Elizondo et al., 2005). The cloning of $\operatorname{trp} m 7$ from this new allele of touchdown revealed a nonsense mutation that truncates TRPM7 before the pore and kinase domains. Given that zebrafish embryos develop externally, and that touchdown mutants are viable into early juvenile stages, we used this new allele of touchdown to explore the putative roles for TRPM7 in vivo. One 

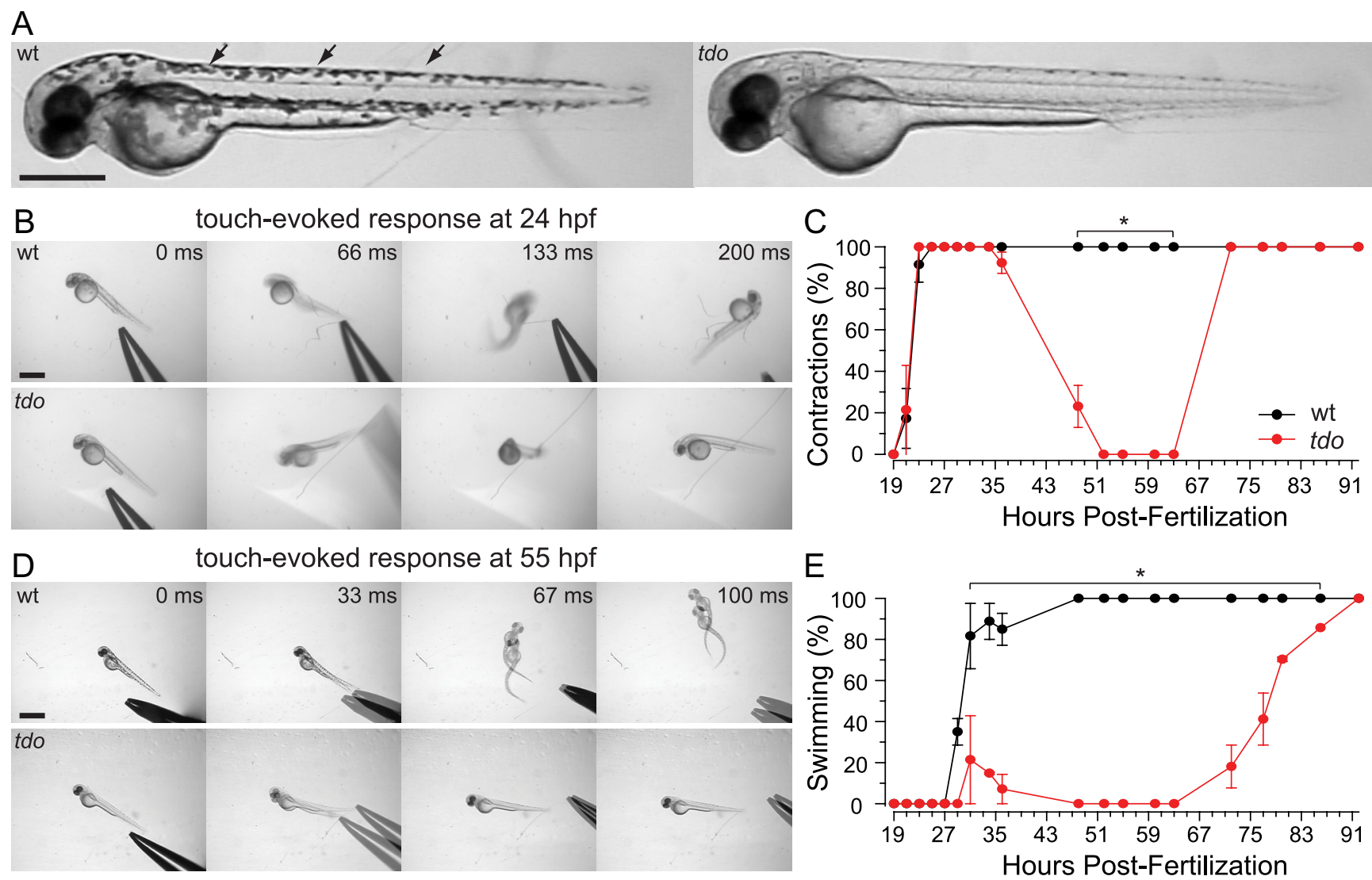

Figure 1. touchdown mutants are hypopigmented and display a transient loss of touch-evoked behaviors. $A$, Lateral view of wild-type (left) and touchdown mutant (right) larvae at 55 hpf. Arrows point to a few of the visible melanophores, which contribute to pigmentation in wild-type zebrafish but are missing in touchdown larvae. Scale bar, $200 \mu \mathrm{m}$. $\boldsymbol{B}$, Frames of videos showing touch-evoked escape contractions at $26 \mathrm{hpf}$ of wild-type (top) and touchdown (bottom) embryos. $0 \mathrm{~ms}$ is the video frame preceding contact of the embryo by the stimulating probe. Scale bar, 500 $\mu \mathrm{m}$. C, The percentage of wild-type (black) and touchdown mutants (red) displaying touch-evoked contractions between 19 and $92 \mathrm{hpf}$ ( $n=24$ from three clutches) showing that touchdown mutants do not respond between 52 and $63 \mathrm{hpf}$. Values represent the average $\pm \mathrm{SEM}$. ${ }^{*} p<0.05, t$ test. $\boldsymbol{D}$, Video frames showing touch-evoked swimming at $55 \mathrm{hpf}$ of wild-type (top) but not of touchdown (bottom) larvae. Of note, swimming larvae sometimes appear in two places as the behavior was faster than the sampling rate of the camera. Scale bar, $500 \mu \mathrm{m}$. $\boldsymbol{E}$, The percentage of wild-type (black) and touchdown mutants (red) displaying touch-evoked swimming between 19 and $92 \mathrm{hpf}$ ( $n=24$ from three clutches).

possibility consistent with our findings is that TRPM7 channel activity contributes to transmitter release at central synapses, similar to that proposed for mammalian TRPM7 at peripheral synapses.

\section{Materials and Methods}

Materials. All reagents were obtained from Sigma-Aldrich. NMDA (100 $\mathrm{mm}$ ) and D-tubocurarine (curare; $15 \mathrm{~mm}$ ) were diluted from the indicated stock concentration.

Animal care and use. Zebrafish were bred and raised according to approved guidelines set forth by the University Committee on Use and Care of Animals, University of Michigan and the Animal Experimentation Ethics Committee, Université de Montréal. The ssx-mini-ICP:eGFP stable transgenic line and zCREST2 enhancer element was provided by Dr. Hitoshi Okamoto (RIKEN, Wako-city Saitama, Japan). Touchdown alleles $t d o^{b 508}$ and $t d o^{t z 310 c}$ were provided by Dr. Paul Henion (Ohio State University, Columbus, $\mathrm{OH}$ ) and The Zebrafish International Resource Center (P40 RR12546 from NIH-NCRR), respectively. Touchdown allele $t d o^{m i 174}$ was identified in a screen conducted at the University of Michigan, Ann Arbor following standard procedures (Haffter and NüssleinVolhard, 1996).

Behavioral analysis. Before the beginning of an experiment, embryos of indeterminable sex were dechorionated with Pronase, developmental staged as described previously (Kimmel et al., 1995), and placed individually into the chambers of 24 -well plates. Touch-evoked behaviors were elicited by touching an embryo/larvae up to three times with a pair of No. 5 forceps between 19-92 h postfertilization (hpf). Behaviors were re- corded at $15-30 \mathrm{~Hz}$ using a Panasonic CCD camera (wv-BP330) mounted on a S6E stereomicroscope (Leica Microsystems). Images were captured with PRG Flycap (Point Gray), and analyzed off-line using Image (NIH).

Molecular biology. 5' and 3' RACE-ready cDNA (GeneRacer kit; Invitrogen) was synthesized from total RNA extracted from the WIK genetic background at $60 \mathrm{hpf}$ using TRIzol (Invitrogen). Cloning of trpm 7 was performed by PCR amplification (Pfu Turbo Polymerase; Stratagene) using RACE primers and the following gene-specific primers: forward, 5'-GTGAAGTCGATCTCTAACCTGGT-3'; and reverse, 5' -TAGGATGAAGTGATAGCGCTGATA-3'.

The PCR product was A-tailed using TaqDNA Polymerase and subcloned into pGEM-Teasy (both from Promega). Five individual clones were sequenced and compared. An endogenous XhoI site was removed from the $\operatorname{trpm} 7$ coding sequence by base pair substitution at position 3258 to facilitate future directional subcloning using a QuikChange Site-Directed Mutagenesis kit (Stratagene) and the following primers: top, 5'-GTGTGCGAACTCAAGTGAGGAA-3'; and bottom, 5' -TTCCTCACTTGAGTTCGCACAC- $3^{\prime}$. The underlined base pair indicates the position of the substitution. Assembly of the full-length trpm7 cDNA was achieved via splice-by-overlap extension using the trpm7specific primers listed above in conjunction with the following primers: forward, 5' -GGTACCATGTCCCAGAAGTCCTGG-3'; and reverse, 5'-CTCGAGTCACAGCATTAAGCGCAT-3'. The underlined sequences denote the inclusion of KpnI and XhoI sites for directional subcloning into pcDNA3.0 (Invitrogen). To clone mutant trpm7, total RNA from homozygous mutants was extracted as above, followed 
by reverse transcription using an oligo(dT) primer and SuperScript III according to manufactures guidelines (Invitrogen). Full-length mutant $\operatorname{trpm} 7$ was assembled by splice-by-overlap extension, as above. Five individual clones were sequenced and compared. A fulllength functional cDNA encoding TRPM6 (accession number HQ261760) was isolated by RACE using the following primers raised against the zebrafish expressed sequence tag AL915621: forward, 5' CAACAAGGAACTGCTAGTGCTGGAT-3'; and reverse: 5'-AAAGGCAGGAACAGACTTCTTCAGG-3' .

Mutations within $\operatorname{trpm} 7$ were generated using the QuikChange SiteDirected Mutagenesis kit and are referred to using the one letter codes, such that D1686A represents the replacement of aspartate at position 1686 with an alanine.

The rescue plasmid was generated by substitution of eGFP's initial methionine in pEGFP-1 (Clontech) containing the hsp70 promoter and zebrafish trpm7's stop codon with EcoR V sites. Zebrafish $\operatorname{trpm} 7$ was then subcloned into pEGFP-1 between KpnI and EcoR V. The zCREST2 enhancer (Uemura et al., 2005) was PCR amplified using the following primers: forward, 5'-AACTCGAGGTGCAGCTTTAGACATTTA-3'; and reverse, 5'-AAAGCTTGAATTCCAGCACCATAATT-3'. The underlined sequences denote the inclusion of XhoI and HindIII sites. PCR product was digested with XhoI and HindIII and directionally subcloned into the hsp70:TRPM7-eGFP to generate zCREST2-hsp70:TRPM7-eGFP. All constructs were confirmed by sequencing (DNA Sequencing Core, University of Michigan).

In situ hybridization and immunohistochemistry. In situ hybridization was performed using previously published procedures (Westerfield, 2000), with some modifications. In brief, embryos were reared in 1-phenyl-2-thiourea beginning $\sim 20 \mathrm{hpf}$ to prevent pigmentation, and fixed with 4\% paraformaldehyde in PBS for $1 \mathrm{~h}$ at room temperature $\left(22^{\circ} \mathrm{C}\right)$. Embryos were then dehydrated with an increasing percentage of methanol in PBS, and stored at $-20^{\circ} \mathrm{C}$ for $30 \mathrm{~min}$ in methanol. Thereafter, embryos were rehydrated by decreasing the percentage of methanol in PBS containing $0.1 \%$ Tween, incubated at $37^{\circ} \mathrm{C}$ with proteinase $\mathrm{K}$ for $15 \mathrm{~min}$ to increase the penetration of riboprobes, and fixed again for 30 $\mathrm{min}$ at room temperature. Template for probe synthesis was generated by PCR using commercially available T7 and SP6 primers (Invitrogen). Full-length antisense and sense control Digoxigenin (DIG) riboprobes were synthesized from PCR product and hydrolyzed to $\sim 1 \mathrm{~kb}$. Probe integrity was first assessed by gel electrophoresis and then bound for $\sim 16$ $\mathrm{h}$ at $65^{\circ} \mathrm{C}$. Preblocked anti-DIG antibody conjugated to alkaline phosphatase (Roche Applied Science) was incubated at room temperature for $4 \mathrm{~h}$ followed by chromogenic detection using a NBT/BCIP solution (Roche Applied Science). The chromogenic reaction was quenched at various time points for both antisense and sense control conditions. Staining by the antisense probe was considered specific when staining was absent from tissue treated with the sense control under the same conditions.

Labeling of eGFP conjugated to TRPM7 (TRPM7-eGFP) or from the stable transgenic line ssx-mini-ICP:eGFP was performed using standard lab procedures at room temperature $\left(22^{\circ} \mathrm{C}\right)$. In brief, embryos were fixed in $4 \%$ paraformaldehyde for $30 \mathrm{~min}$ and then washed five times for 10 min each in wash buffer (PBS containing $0.1 \%$ Triton X-100) and twice for $10 \mathrm{~min}$ in blocking buffer (wash buffer containing $2 \mathrm{mg} / \mathrm{ml}$ bovine serum albumin). Rabbit anti-GFP primary antibody (Torrey Pines Biolabs) was diluted $1 / 1000$ in blocking buffer and bound overnight. Next, embryos were washed five times for $10 \mathrm{~min}$ in wash buffer, two times for 10 min in blocking buffer, and then incubated in blocking buffer containing secondary antibody (anti-rabbit Alexa-488; Invitrogen) at 1/1000 for $4 \mathrm{~h}$. Embryos were then washed 10 times in wash buffer for $10 \mathrm{~min}$ each and mounted in $70 \%$ glycerol diluted with PBS. Images were captured with a Leica TCS laser scanning confocal microscope. Rohon-Beard (RB) cell number was assayed by counting RBs from three somites above the anal opening. The images of TRPM7-eGFP-positive RB cell bodies were processed with the Blur function of Adobe Photoshop CS4 to average adjacent pixels. The images of the eGFP-positive RB neurites were also optimized using the Autocontrast function of Adobe Photoshop.

Rescue plasmid and morpholino injections. Plasmid-based rescue of touch-evoked behaviors in $t d o^{\text {mi174 }}$ mutants was performed by injecting
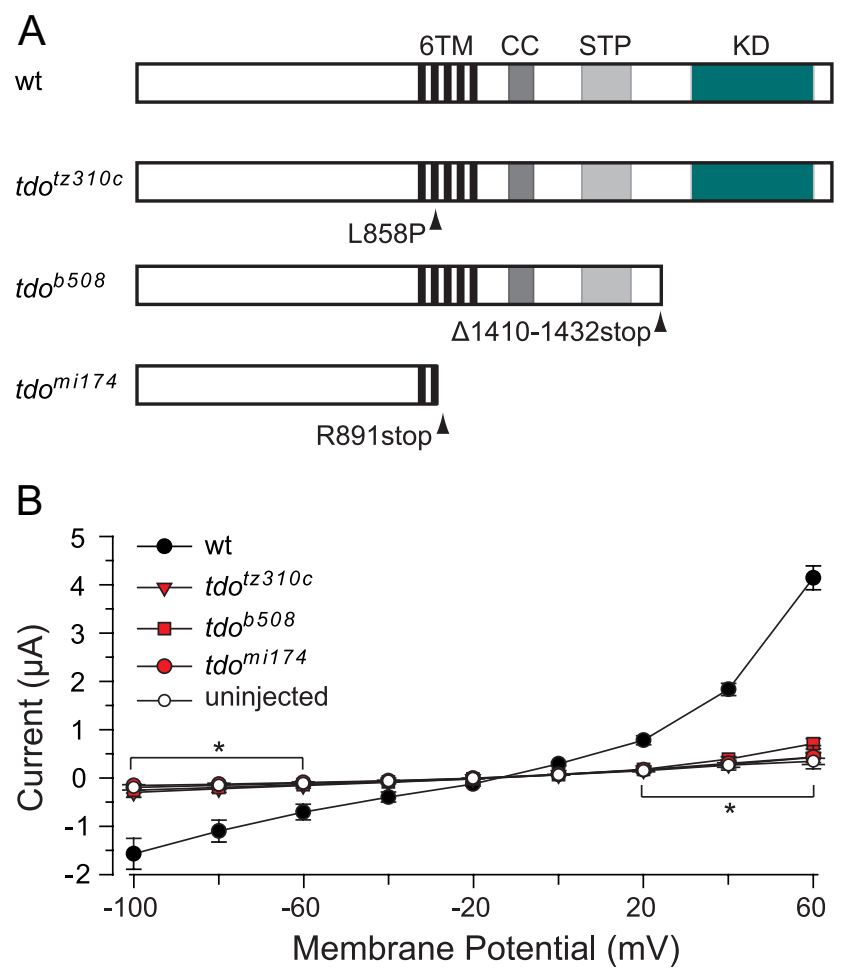

Figure 2. touchdown alleles $t d 0^{t z 310 c}, t d 0^{b 508}$, and $t d 0^{m i 174}$ harbor mutations that abolish channel activity. $\boldsymbol{A}$, A graphic representation of TRPM7 indicating the location of mutations in the three alleles of touchdown. CC, Coiled-coil domain; STP, serine-threonine-proline rich domain; KD, kinase domain. $B$, Average current responses of oocytes expressing wild-type TRPM7 or touchdown alleles to voltage ramps between -100 and $+60 \mathrm{mV}$ ( $n=10$ oocytes for each allele) showing that all three alleles lack channel activity (i.e., the currents in response to ramps were not significantly different from those of uninjected oocytes). Values represent the average \pm SEM. ${ }^{*} p<0.05, t$ test.

$50 \mathrm{pg}$ of the rescue plasmid zCREST2-hsp70:TRPM7-eGFP (or TRPM7 point mutants derived from this plasmid) diluted in $5.0 \mathrm{nl}$ of water containing $0.01 \%$ phenol red into freshly fertilized embryos from $t d o^{\text {mi174 }}$ carrier incrosses at the $1-2$ cell stage using a Nanoinject II system (Drummond Scientific). Embryos were screened at $\sim 24-27 \mathrm{hpf}$ for expression of eGFP in cells consistent with sensory neurons. eGFP-positive embryos were placed individually into 24 -well plates and screened for touch responsiveness at $54 \mathrm{hpf}$. Homozygous touchdown mutants were identified by their hypopigmentation as the rescue plasmid failed to rescue melanocytes. At this time point, touchdown mutants rarely responded to touch $(<1 \%)$, so all hypopigmented larvae that displayed escape contractions, or escape contractions plus a swimming episode of at least one full body length in response to $<4$ tactile stimuli delivered to the tail were scored as "rescued."

The following antisense oligonucleotides (morpholino) were raised against the translational start site of $\operatorname{trpm} 6$ and $\operatorname{trpm} 7$, along with the standard mismatch control morpholino (Gene Tools): trpm6, 5'-ACATGTCTCCTCAAATCTCCTTGAC-3'; $\operatorname{trpm} 7,5^{\prime}$-CCAGGACTTCTGGGACATTCTCTTC-3'; and control, 5'-CCTCTTACCTCAGTTACAATTTATA-3'. The underlined areas indicate the complementary sequences to the initial methionines of trpm6 and trpm7. Morpholinos $(5.0 \mathrm{nl}$ at $200 \mu \mathrm{M})$ were injected as above and sorted for uptake of dye $\sim 6 \mathrm{~h}$ later. Hypopigmentation and responsiveness to touch were assayed between 19-92 hpf.

Recording methods. Two-electrode voltage-clamp recordings from Xenopus oocytes were made with a TurboTec 3 amplifier (NPI Electronics) at room temperature. Recordings were low-pass filtered at $20 \mathrm{~Hz}$ and sampled at $50 \mathrm{~Hz}$. Data acquisition was controlled by pClamp 9 software using a Digidata 1320A interface (Molecular Devices), and solution flow $(\sim 1 \mathrm{ml} / \mathrm{min}$ ) was controlled by a BPS- 8 solution exchange system (ALA Scientific Instruments). In most experiments, the normal oocyte recording solution (ORS) contained the following (in mM): $90 \mathrm{NaCl}, 1 \mathrm{KCl}, 1$ 
A

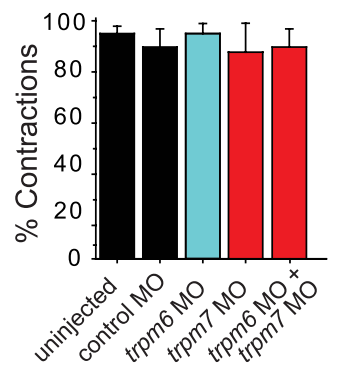

B

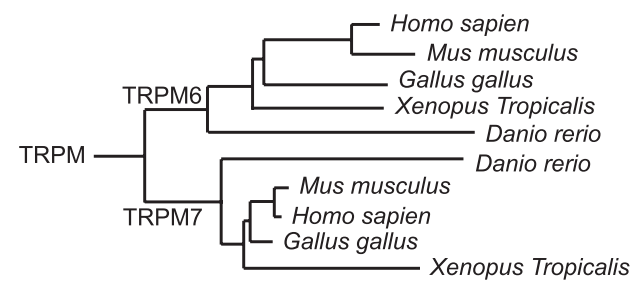

C

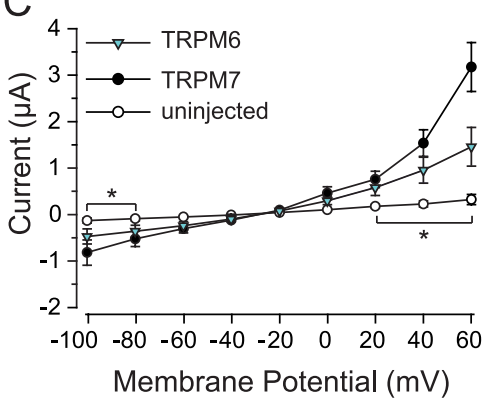

Figure 3. The touchdown phenotype results from the loss of TRPM7 during larval development. $A$, Histogram showing the percentage of embryos injected with the indicated antisense M0 that responded to tactile stimuli with escape contractions at $24 \mathrm{hpf}$ ( $n>60$ from three clutches). Injection of trpm7, trpm6, and trpm $7+$ trpm6 antisense M0s did not affect touch-evoked contractions. $B$, A phylogenetic tree showing the assignment of the identified protein as the zebrafish ortholog of TRPM6. C, Average current responses of oocytes expressing wild-type TRPM7 or TRPM6 to voltage ramps between -100 and $+60 \mathrm{mV}$ ( $n=10$ for each allele), showing that TRPM6 exhibits a outwardly rectifying current similar to TRPM7. *, Current values of TRPM6 and TRPM7 that were significantly different from uninjected controls $(p<0.05)$. The amplitude of TRMP6 responses at $+60 \mathrm{mV}$ was significantly smaller than TRPM7 responses.
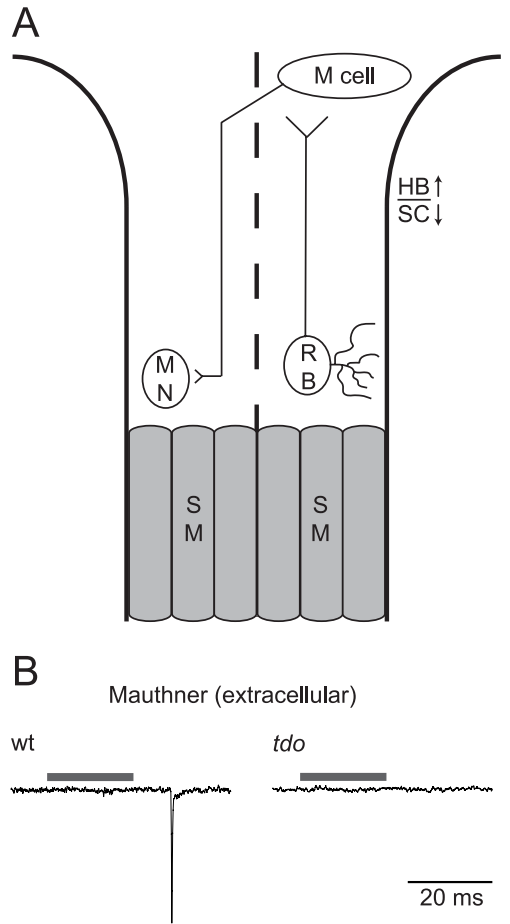

C

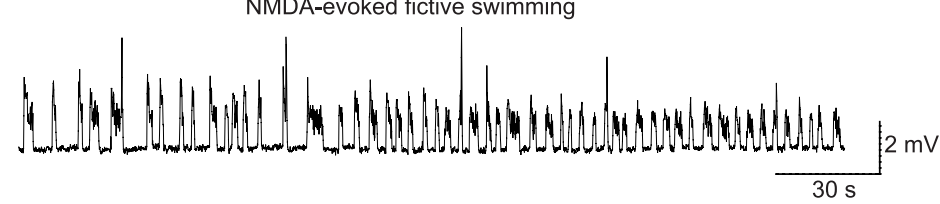

D

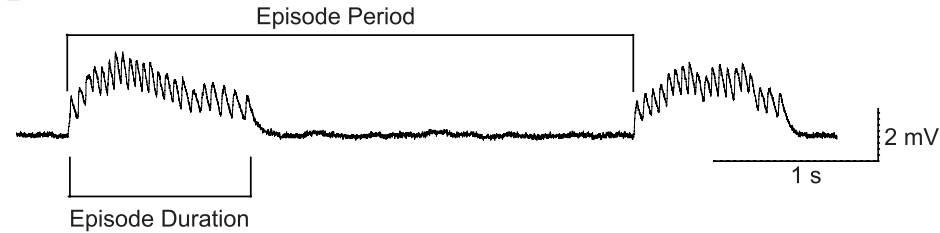

E

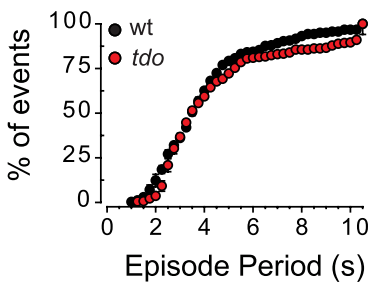

$\mathrm{F}$

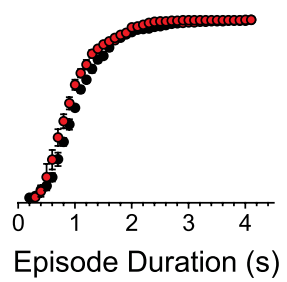

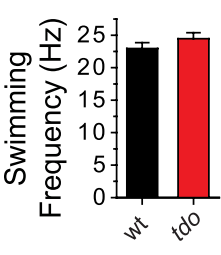

Figure 4. Tactile stimuli in touchdown mutants fails to activate second-order neurons and the locomotor network that generates swimming in zebrafish. $A$, Schematic of a minimal touch-evoked circuit in zebrafish. MN, Motor neuron; SM, skeletal muscle; $\mathrm{HB}$, hindbrain; $\mathrm{SC}$, spinal cord. Dashed line designates the dorsal midline; anterior is up. $\boldsymbol{B}$, Extracellular recordings from wild-type and touchdown mutant larva using electrodes positioned to detect Mauthner neuron spikes showing that tactile stimulation activates $\mathrm{M}$ cells in wild-type but not touchdown larvae at $55 \mathrm{hpf}$. Gray bars indicate time of the stimulus to the puffer pipette. C, Several minutes of NMDA-evoked fictive swimming episodes recorded from axial skeletal muscle in a wild-type larva (55 hpf) in the presence of $3 \mu \mathrm{m}$ curare, a concentration sufficient to attenuate membrane depolarization below the level necessary to induce excitation-contraction coupling. D, A faster sweep of NMDA-evoked fictive swimming detailing episode period, episode duration, and swimming frequency (frequency of endplate potentials within an episode). The first episode lasted $1.06 \mathrm{~s}$ and had 22 peaks, for a swim frequency of $20.75 \mathrm{~Hz}$. E, Cumulative plots of episode periods and durations in wild-type and touchdown mutant larvae showing that episode period and durations are not affected by the mutation. $\boldsymbol{F}$, Histogram showing that the frequency of NMDA-evoked fictive swimming is comparable between wild-type and touchdown mutant larvae $(n=5$ for each).

$\mathrm{MgCl}_{2}, 2 \mathrm{CaCl}_{2}$, and $10 \mathrm{HEPES}$, adjusted to $\mathrm{pH} 7.5$ with $\sim 8 \mathrm{~mm} \mathrm{NaOH}$. In the experiments shown in Figure 6, either a modified ORS that lacked calcium and had $1.3 \mathrm{~mm} \mathrm{MgCl}_{2}$, or a high magnesium-containing solution where the $90 \mathrm{~mm} \mathrm{NaCl}$ was replaced with $60 \mathrm{~mm} \mathrm{MgCl}_{2}$ was used. Borosilicate glass electrodes were broken manually to $\sim 0.5 \mathrm{M} \Omega$ when filled with $3 \mathrm{~m} \mathrm{KCl}$. Oocytes were injected with capped RNA (25 ng) encoding zebrafish TRPM6, TRPM7 ${ }_{\text {WT }}$, TRPM7 $7_{\text {R891stop }}$, TRPM7 $7_{\text {L858P }}$, TRPM7 ${ }_{1410-1432 \text { stop }}$, TRPM7 $_{\text {E1026K }}$, TRPM7 $_{\text {E1026O }}$, and TRPM7 $7_{\mathrm{D} 1686 \mathrm{~A}}$ using a Nanoinject II, and were maintained in Barth's solution at $17^{\circ} \mathrm{C}$ for $\sim 72 \mathrm{~h}$ before electrophysiological characterization. To quantify the outward rectification of wild-type and TRPM $7_{\mathrm{E} 1026 \mathrm{O}}$-expressing oocytes in normal recording solution, we measured the amplitude of the currents at
-100 and $+60 \mathrm{mV}$, as this provided approximately equal driving force in both directions for wild-type TRPM7 (average reversal potential of $-18.0 \pm 1.0 \mathrm{mV}, n=9)$ and so allowed us to calculate a rectification index that increases with the amount of outward rectification by dividing the outward current by the absolute value of the inward current.

Whole-cell recordings from 55-60 hpf zebrafish larvae were made at room temperature using an Axopatch 200B amplifier (Molecular Devices) low-pass filtered at $1-5 \mathrm{kHz}$ and sampled at $1-10 \mathrm{kHz}$. Data acquisition was controlled by pClamp 10 software using a Digidata $1440 \mathrm{~A}$ interface. Recording from axial skeletal muscle and neurons were obtained using previously described procedures (Drapeau et al., 1999; Buss and Drapeau, 2000; Low et al., 2010a,b). In brief, larvae were anesthetized 
A

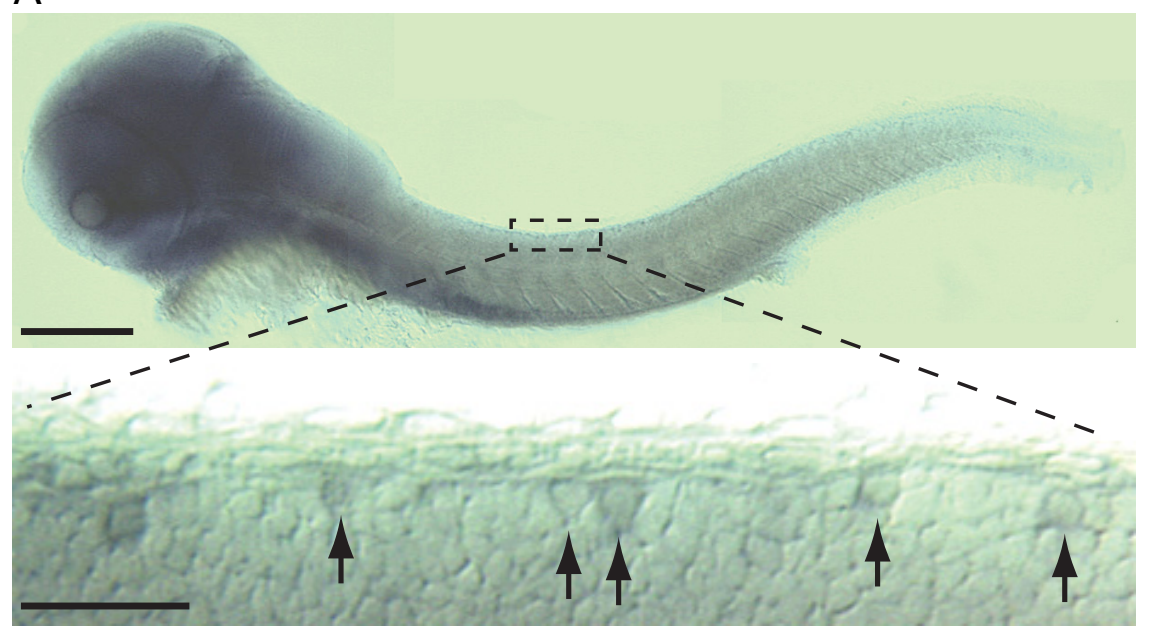

B

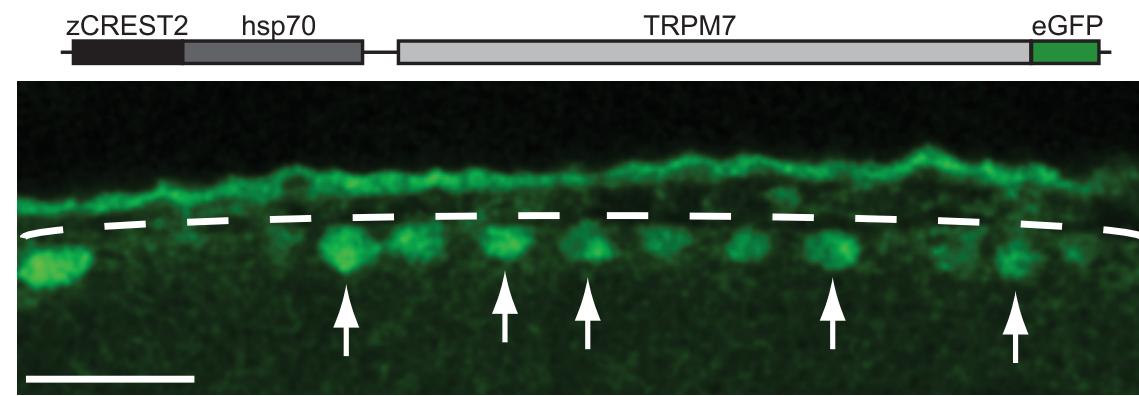

C

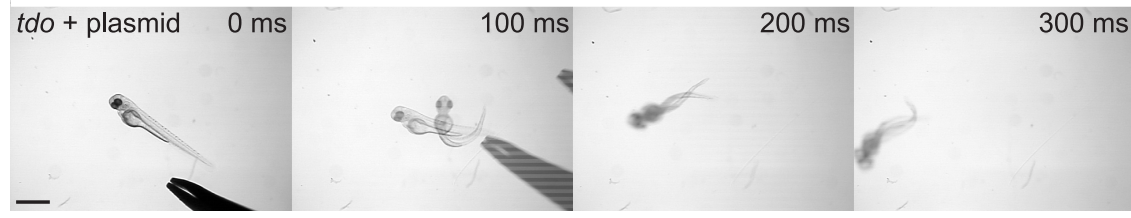

Figure 5. Selective restoration of TRPM7 expression in sensory neurons is sufficient to restore touch-evoked responses. $A$, Top, Lateral view of a $55 \mathrm{hpf}$ larva labeled with a riboprobe against trpm 7 showing that trpm 7 is expressed widely in the larva. Boxed area indicates enlarged area shown below. Scale bar $200 \mu \mathrm{m}$. Bottom, Enlarged area highlighting a few RB sensory neurons expressing trpm7, with some RBs indicated with arrows. Scale bar, $50 \mu \mathrm{m}$. $\boldsymbol{B}$, Top, Rescue plasmid comprised of the sensory neuron enhancer/promoter construct zCREST2-hsp70:TRPM7-eGFP. Bottom, Lateral view of the dorsal spinal cord following injection of the sensory enhancer/promoter construct labeled with anti-GFP showing that TRPM7-eGFP is expressed by many RBs, some of which are marked by arrows. Dashed line denotes the dorsal boundary of the spinal cord. Green labeling above dashed line was also observed in uninjected embryos, indicating that is background labeling of the skin by anti-GFP. Scale bar, $50 \mu \mathrm{m}$. C, Expression of TRPM7 selectively in RBs rescues touch-responsiveness in touchdown mutant larvae. Video frames of touch-evoked swimming from a 55 hpf hypopigmented touchdown mutant expressing the rescue plasmid. $0 \mathrm{~ms}$ is the video frame preceding contact of the embryo by the stimulating probe. Scale bar, $500 \mu \mathrm{m}$.

in Evans solution containing the following (in $\mathrm{mm}$ ): $134 \mathrm{NaCl}, 2.9 \mathrm{KCl}$, $2.1 \mathrm{CaCl}_{2}, 1.2 \mathrm{MgCl}_{2}, 10$ glucose, 10 HEPES, pH 7.8 with $\mathrm{NaOH}$, supplemented with $0.02 \%(\mathrm{w} / \mathrm{v})$ tricaine. The skin of a larva pinned to a $35 \mathrm{~mm}$ Sylgard-coated dish was scored with a broken pipette and removed with a pair of No. 5 forceps. The external recording solution was Evans supplemented with curare ( $3 \mu \mathrm{M}$ for muscle, $15 \mu \mathrm{M}$ for neuron recordings) and was perfused over the prep throughout the recording session at $\sim 1$ $\mathrm{ml} / \mathrm{min}$. To make neuron recordings, the bath solution was transiently replaced with recording solution containing $2 \mathrm{mg} / \mathrm{ml}$ collagenase type XI and incubated until the muscle started to separate. The muscle was then peeled away using suction applied from a broken pipette $(\sim 50 \mu \mathrm{m})$. The electrode solution for both muscle and neuron recordings contained the following (in $\mathrm{mm}$ ): $116 \mathrm{~K}$-gluconate, $16 \mathrm{KCl}, 2 \mathrm{MgCl}_{2}, 10 \mathrm{HEPES}, 10$ EGTA, at pH 7.2 with $\mathrm{KOH}$, and $0.1 \%$ Sulforhodamine B for cell type identification. Borosilicate glass electrodes had resistances of 6-10 M $\Omega$ and 10-14 $\mathrm{M} \Omega$ for muscle and neuron recordings, respectively.
Extracellular recordings of Mauthner cell activity were made with borosilicate glass electrodes filled with external recording solution that had resistances of $\sim 1 \mathrm{M} \Omega$. The signal was amplified using the Axopatch 200B. The recording electrode was always positioned just above the caudal portion of the otolith, ipsilateral to the side of stimulation, which was shown to give the largest responses in $96 \mathrm{hpf}$ fish (Prugh et al., 1982). These authors, however, demonstrated that Mauthner spikes could be detected at all electrode positions tested, including in a small water drop with a free-moving fish nearby. Thus, the precise positioning of the recording electrode in the 55-60 hpflarvae was unlikely to have been critical. Tactile stimuli were delivered via pulses of bath solution to the head, yolk sac, or tail regions via a broken pipette $(\sim 20 \mu \mathrm{m})$. The pressure $(20 \mathrm{psi})$ and duration $(50 \mathrm{~ms})$ of the stimulus was controlled by a Picospritzer II (Parker Hannifin).

NMDA-evoked fictive swimming was achieved by perfusing the bath with recording solution containing $100 \mu \mathrm{M}$ NMDA. Electrical and tactile stimulation of peripheral neurites was achieved by placing a bipolar probe (Model 2100; A-M Systems) controlled by piezoelectric stimulator (Model PCS-1000 with a PCS-250 patch-clamp driver; EXFO Life Sciences) upon eGFP-positive neurites $\sim 100 \mu \mathrm{m}$ from the cell body. For all recording methods, the initial data analysis was done with Clampfit 10; figures were prepared using Sigma Plot 11.0 and Adobe Illustrator CS5.

Statistical analysis. Data were analyzed using Microsoft Excel or Sigmaplot (versions 9-11). In text and in all figures, data are presented as the average \pm SEM.

\section{Results}

\section{mi174 is a new allele of the transiently} touch-unresponsive/hypopigmented mutant touchdown

During early development, zebrafish display two highly stereotyped touch-evoked escape behaviors: contractions and swimming (Saint-Amant and Drapeau, 1998). Touch-evoked contractions begin at $\sim 21$ hpf and are characterized by one to three rapid, alternating contractions of the trunk and tail toward the head in response to tactile stimuli delivered along the body axis. Touch-evoked swimming capable of propelling embryos forward at least one body length begins at $\sim 27 \mathrm{hpf}$.

In a forward genetic screen for zebrafish mutants that displayed abnormal touch-evoked escape behaviors, one family, mi174, was found to possess a recessive mutation resulting in offspring that were hypopigmented (Fig. 1A) and transiently touch unresponsive between 52-63 hpf (Fig. $1 B-D$ ). Considering that hypopigmentation is an easily observable phenotype, we reasoned that mi174 might represent a new allele of a previously identified mutant. Therefore, complementation analysis was performed with touchdown ( $t d o^{t z 310 c}$ ) and touchtone $\left(t c t^{b 508}\right)$, two previously identified touch-unresponsive/hypopigmented mutants (Granato et al., 1996; Arduini and Henion, 2004). Pairwise 
crosses between mi174, touchdown, and touchtone failed to complement, indicating that all were allelic. As touchdown was the first allele in this series of mutants to be reported (Granato et al., 1996), alleles will herein be referred to as alleles of touchdown $(t d o)$, following established guidelines (Zebrafish Information Network).

$t d o^{t z 310 c}, t d o^{b 508}$, and $t d o^{m i 174}$ harbor

mutations in $\operatorname{trpm} 7$ that abolish

channel activity

An allele of touchdown $\left(t d o^{b 508}\right)$ was shown to harbor a mutation in the gene encoding TRPM7 (Elizondo et al., 2005), one of two members of the TRP superfamily of cation channels that possess enzymatically active kinases on their C termini (Montell, 2005). Therefore, trpm7 was cloned from touchdown alleles $t d o^{\text {mi174 }}$ and $t d o^{t z 310 c}$, which revealed a nonsense mutation in the intracellular loop between Transmembrane Domains (TM)2 and TM3 (TRPM7 R891stop ), and a missense mutation within TM2 (TRPM7 ${ }_{\text {L858P }}$ ), respectively (Fig. $2 \mathrm{~A}$ ). To determine the consequence of these mutations, along with the frameshift-truncating mutation found in touchdown allele $t d o^{b 508}$ (TRPM7 $1410-1432$ stop $)$ (Elizondo et al., 2005), TRPM7 channel activity was examined under two-electrode voltage clamp in Xenopus oocytes. Oocytes injected with RNA encoding wild-type TRPM7 were found to exhibit an outwardly rectifying current when subjected to voltage ramps (Fig. $2 B$ ). In contrast, oocytes injected with RNA encoding TRPM7 $7_{\text {R891stop }}$, TRPM7 $_{\text {L858P }}$, or TRPM7 $1410-1432$ stop failed to generate currents greater than those of uninjected oocytes, indicating a loss of TRPM7 channel activity in these three alleles of touchdown. These findings are consistent with the previously reported activity of wild-type mammalian TRPM7 expressed in Xenopus oocytes (Chubanov et al., 2004) and loss of channel activity following deletion of the $\mathrm{C}$ terminus of TRPM7 (Matsushita et al., 2005). Of note, touchdown allele $t d o^{\text {millt }}$ was chosen for subsequent experiments, as it represented the most severely truncated allele in the series of touchdown mutants.

\section{The touchdown phenotype results from the loss of larval TRPM7}

A possible explanation for the lack of a phenotype at $24 \mathrm{hpf}$ in touchdown mutants is that TRPM7 is required for tactile responses at all developmental stages, but early in development, sufficient wild-type maternal RNA transcripts or protein are inherited to transiently compensate for nonfunctional TRPM7 made by touchdown mutants. Unfortunately, commercially available antibodies for mammalian TRPM7 failed to recognize zebrafish TRPM7, and therefore we were not able to test whether maternally inherited TRPM7 protein was present in the early larvae. However, a trpm 7 transcript was identified by RT-PCR from RNA obtained at the one-cell stage, a time point preceding the initiation of zygotic transcription. To determine whether the maternally inherited $\operatorname{trpm} 7$ RNA accounted for the early period of touch responsiveness in zebrafish, an antisense [morpholino oligonucleotide (MO)] against trpm7 was injected into wild-type embryos at the one-cell stage, which should block the translation of both the maternal and zygotic mRNAs. We found that TRPM7 MO-injected wild-type embryos responded to touch with escape contractions at $24 \mathrm{hpf}$ (Fig. $3 \mathrm{~A}$ ), but were unresponsive to touch at $55 \mathrm{hpf}$. Thus, the TRPM7 MO reproduced the touchdown mutant phenotype, which suggests that maternally inherited wildtype $\operatorname{trpm} 7$ transcripts do not account for the early period of touch responsiveness in touchdown mutants.

Within the TRP family, TRPM6 is most similar to TRPM7 and is the only other TRP channel with an enzymatically active kinase on its $\mathrm{C}$ terminus. We therefore tested whether TRPM6 was necessary for the early touch-evoked contractions present in touchdown mutants. A zebrafish TRPM6 cDNA that encoded an 1875 aa peptide with 70\% conserved sequence similarity to mouse TRPM6 was identified (Fig. 3B). RNA encoding TRPM6 produced currents similar to zebrafish TRPM7 when expressed by oocytes (Fig. 3C). Wild-type embryos injected with an antisense morpholino oligonucleotide directed against TRPM6 (trpm6 MO), which blocked the production of TRPM6 currents in oocytes coinjected with trpm6 RNA and the antisense MO, responded to tactile stimuli at 24 hpf with contractions similar to uninjected and control morpholino-injected embryos (Fig. 3A). Furthermore, all trpm6 MO-injected embryos responded to touch at $55 \mathrm{hpf}$ with swimming ( $n>60$ from three clutches). Thus, TRPM6 does not appear to be essential for touch-evoked escape behaviors during the first $3 \mathrm{~d}$ of development. 
TRPM7 is required for the activation of interneurons and the zebrafish locomotor network

To gain insight into how the loss of TRPM7 resulted in the transient loss of touch-evoked escape behaviors, we explored activity within the touch-evoked locomotor network of touchdown mutants electrophysiologically. During the first $3 \mathrm{~d}$ of zebrafish development, tactile stimuli to the trunk or tail is detected by mechanosensitive RBs, which are a transient population of sensory neurons located within the dorsal spinal cord (Fig. 4A). These mechanosensitive neurons project axons into the hindbrain where they lead to the activation of $\sim 90$ bilateral pairs of reticulospinal neurons during motor behaviors (Gahtan et al., 2002). Among these reticulospinal neurons, the role of the Mauthner $(\mathrm{M})$ cells in fish are best understood (Korn and Faber, 2005). M cells receive direct sensory input from mechanosensitive neurons (Zottoli and Faber, 1979) and in turn make monosynaptic contacts with motor neurons (Jontes et al., 2000), which are likely excitatory (Mongeon et al., 2008). Thus RBs, M cells, and motor neurons represent a three-neuron circuit capable of relaying tactile stimuli to muscle. Tactile stimulation of the head, tail, and yolk sac was found to evoke $\mathrm{M}$ cell activity in eight of 15 wild-type larvae studied at 55-60 hpf (Fig. 4B). The activation of $M$ cells in only a subset of the larvae examined was expected, as tactile stimulation of older larvae ( $96 \mathrm{hpf}$ ) gave similar results (Eaton et al., 1984). Both results indicate that non-M cell pathways also mediate escape responses in zebrafish. In contrast to wild-type larvae, similar tactile stimuli failed to evoke activity within any $\mathrm{M}$ cells of touchdown mutants $(n=0 / 15)$.

Although tactile stimuli failed to activate $\mathrm{M}$ cells in touchdown mutants, we examined whether the exogenous activation of the locomotor network in mutants could result in patterned activity. To this end, larvae were bathed in the excitatory amino acid NMDA, which has been shown to induce fictive swimming similar to that elicited by tactile stimuli (Cui et al., 2004). In the absence of NMDA, spontaneous bouts of fictive swimming were very rarely observed (wt, $n=1 / 5$; tdo, $n=0 / 5$ during 10 min of baseline recording). In contrast, the application of NMDA to the bath induced repetitive bouts of fictive swimming within $10 \mathrm{~min}$ in every wild-type and mutant larva tested (wt, $n=5 / 5 ;$ tdo, $n=$ 5/5) (Fig. 4C). A closer examination of NMDA-evoked fictive swimming revealed that the episode period, episode duration, and the frequency of fictive swimming were indistinguishable between wild-type and touchdown mutant larvae (Fig. 4E,F). Therefore, TRPM7 is not required for the formation and/or function of the locomotor network that underlies swimming in zebrafish.

\section{Restoration of TRPM7 within sensory neurons was sufficient} to restore responsiveness to touch

In an attempt to pinpoint the cellular locus of defect in touchdown mutants, the expression pattern of $\operatorname{trp} m 7$ was assessed during the period of touch-unresponsiveness. In situ hybridization revealed that $\operatorname{trpm} 7$ appeared to be expressed at low levels by most cell types at $55 \mathrm{hpf}$ (Fig. 5A), and at higher levels by cells whose location in the spinal cord indicated they were sensory neurons (Bernhardt et al., 1990). Based on the elevated expression of trpm7 by sensory neurons, and the finding that tactile stimuli failed to activate $\mathrm{M}$ cells in touchdown mutants, we sought a way to restore wild-type TRPM7 expression selectively within sensory neurons. In zebrafish transient transgenics, the zCREST2-hsp70 sensory neuron enhancer-promoter construct has been shown to drive the expression of eGFP exclusively in sensory neurons, while in stable transgenics this construct has been observed to
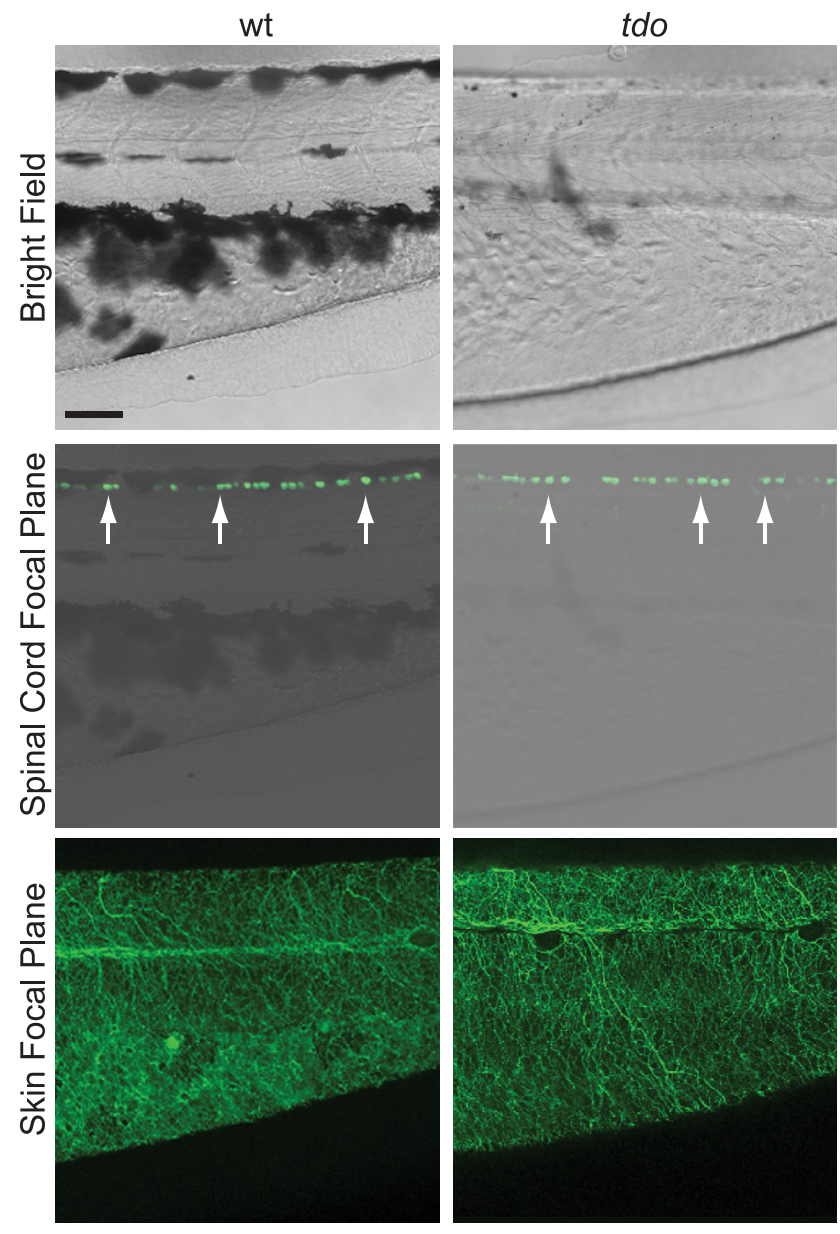

Figure 7. The gross morphology of RBs appears normal in touchdown mutants. Top, A lateral bright field view of the trunk and tail regions from ssx-mini-ICP:eGFP transgenic wild-type and touchdown mutant larvae at $55 \mathrm{hpf}$. Rostral is to the left and dorsal is up. Scale bar, $50 \mu \mathrm{m}$. Middle, Confocal microscope view of the same regions displaying RB cell bodies (detected by using anti-GFP and a fluorescent secondary antibody) superimposed over the bright field image. The microscope was focused at the level of the RB cell bodies. Bottom, The same larvae viewed in a different focal plane that highlights RB peripheral neurites within the skin.

drive eGFP expression within both sensory neurons and motor neurons (Uemura et al., 2005). Therefore, in transient transgenics, we assessed the ability of TRPM7-eGFP under the control of the zCREST2-hsp70 to rescue touch-evoked behaviors in touchdown mutants. Clutches obtained from crosses of heterozygote carriers injected with this rescue plasmid (zCREST2-hsp70: TRPM7-eGFP) (Fig. 5B) exhibited a Mendelian ratio of pigmentation (71.9 $\pm 1.2 \%$ normal: $28.1 \pm 1.2 \%$ hypopigmented; $n>$ 100 larvae from five clutches) and displayed selective expression of eGFP in RB sensory neurons (Fig. $5 B$ ), a finding that confirms the reported sensory neuron specificity of the zCREST2-hsp70 enhancer-promoter construct in transient transgenics. When assayed, $\sim 70 \%$ of hypopigmented mutant larvae injected with the rescue plasmid responded to tactile stimuli at $54 \mathrm{hpf}$ (Fig. 5C), a time point when $<1 \%$ of uninjected mutant larvae exhibited touch-evoked responses (Fig. 6C). Thus, restoration of wild-type TRPM7 selectively in touchdown mutant sensory neurons was sufficient to restore responsiveness to touch.

\section{TRPM7's kinase activity and divalent selectivity are dispensable for touch-responsiveness}

To gain insight into the role of TRPM7 within these sensory neurons, we used the rescue plasmid to assess the ability of mod- 
ified versions of TRPM7 to rescue touch responsiveness in touchdown mutants. Previous work has identified single amino acid substitutions in mammalian TRPM7 that selectively remove the phosphotransferase activity of TRPM7's kinase $\left(\right.$ TRPM7 $\left._{\text {D1686A }}\right)$ (Matsushita et al., 2005) and TRPM7's inward selectivity for divalent cations $\left(\mathrm{TRPM} 7_{\mathrm{E} 1026 \mathrm{Q}}\right.$ ) ( $\mathrm{Li}$ et al., 2007), or abolish TRPM7 channel activity (TRPM7 $_{\text {E1026K }}$ ) (Li et al., 2007). The consequence of these analogous amino acid substitutions in zebrafish TRPM7 were first assessed in oocytes (Fig. 6A). In agreement with studies of mammalian TRPM7, zebrafish TRPM7 D1686A $_{\text {(kinase }}$ mutant)-injected oocytes exhibited TRPM7 currents indistinguishable from wild-type, and TRPM7 $7_{\mathrm{E} 1026 \mathrm{~K}}$ (pore-killing mutant)injected oocytes failed to generate currents above background. Finally, TRPM7 $7_{\mathrm{E} 1026 \mathrm{Q}}$ (pore-selectivity mutant)-injected oocytes exhibited less outward rectification $\left(\mathrm{g}_{+60 \mathrm{mv}} /\right.$ $\mathrm{g}_{-100 \mathrm{mV}}$ TRPM7 $_{\mathrm{WT}}: 6.2 \pm 0.9, n=8$; vs TRPM7 $_{\text {E1026Q }}: 1.4 \pm 0.1, n=9$ ), consistent with a reduction in divalent selectivity. To further compare ion selectivity, oocytes expressing wild-type or TRPM7 $7_{\mathrm{E} 1026 \mathrm{Q}}$ were shifted from the normal high sodium-containing solution ( $98 \mathrm{~mm} \mathrm{Na}, 1.3 \mathrm{~mm} \mathrm{Mg}$ ) into a solution where most of the sodium was replaced with magnesium $(60 \mathrm{~mm} \mathrm{Mg}, 8$ $\mathrm{mm} \mathrm{Na}$ ). The altered recording solution resulted in a small negative shift in the reversal potential of the wild-type-injected oocytes $(\Delta-5.6 \pm 2.1 \mathrm{mV}, n=8)$. In contrast, $\mathrm{TRPM}_{\mathrm{E} 1026 \mathrm{Q}}$-injected oocytes exhibited a large negative shift in the reversal potential $(\Delta-51.9 \pm 11.0, n=7)$, indicating that TRPM7 $7_{\mathrm{E} 1026 \mathrm{O}}$ is much less permeable to magnesium (Fig. 6B).

Injection of rescue plasmids harboring these amino acid substitutions revealed that the TRPM7 $7_{\mathrm{D} 1686 \mathrm{~A}}$ kinase mutant and the TRPM7 $7_{\mathrm{E} 1026 \mathrm{Q}}$ pore-selectivity mutant each rescued touch-evoked responsiveness in touchdown mutants. In contrast, the putative pore-killing mutant TRPM7 $7_{\mathrm{E} 1026 \mathrm{~K}}$ failed to rescue touch-evoked responsiveness in touchdown mutants (see Discussion, below) (Fig. 6C). These experiments indicate that TRPM7's kinase activity and preference for divalent cations are dispensable for touch responsiveness, and represent the first in vivo assessment of the contribution of TRPM7's kinase and ion selectivity in a native cell.

\section{Morphology of sensory neurons appears normal in touchdown mutants}

As mammalian TRPM7 has been linked to cell survival (Nadler et al., 2001; Schmitz et al., 2003), and melanophores die in touchdown mutants (Elizondo et al., 2005), we examined whether zebrafish TRPM7 might be required for cell survival in sensory neurons. As an initial test for sensory neuron cell death in touchdown mutants, we incubated larvae in acridine orange, a vital dye
B

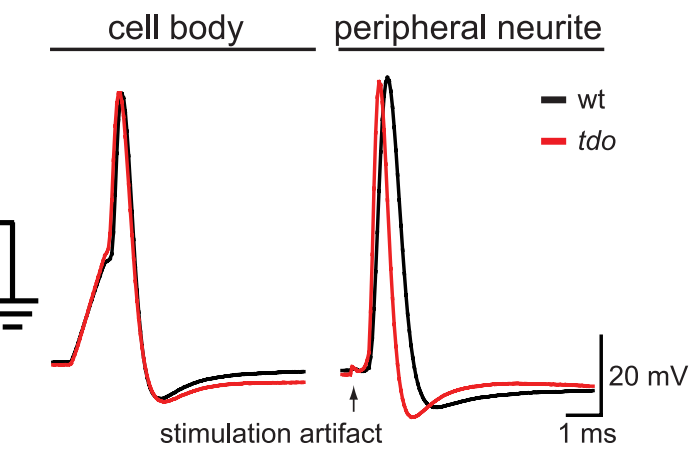

Type II Rohon-Beard
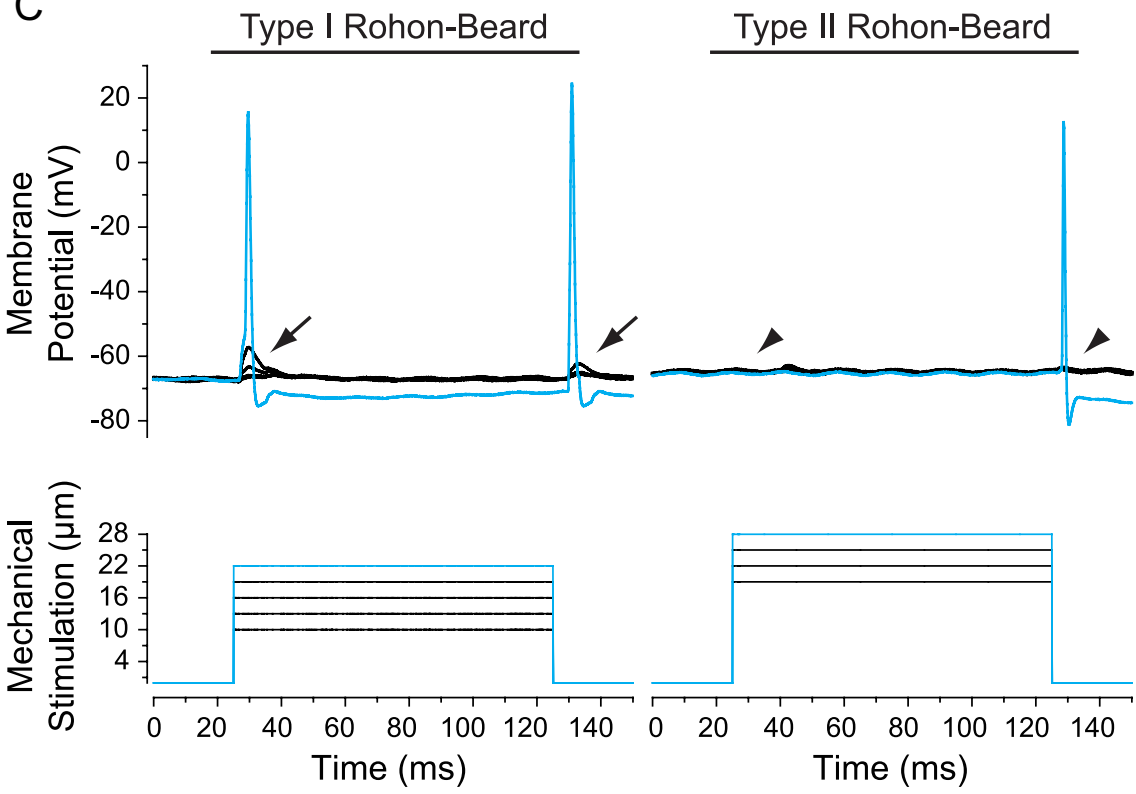

Figure 8. Cutaneous mechanoreceptors respond to tactile stimuli in touchdown mutants. $A$, A representation of the preparation used to record electrical and tactile-evoked responses in RBs. The dashed line designates the dorsal midline. The light gray area indicates the region in which the skin and muscle were removed to allow access to a contralateral RB cell body with an intact (white). The stimulating probe was placed on the skin directly overlying the peripheral neurite. $\boldsymbol{B}, \mathrm{RB}$ responses to depolarizing current injections ( $2 \mathrm{~ms}$ ) to the cell body, and extracellular electrical stimulation (1 ms) of the peripheral neurite showing that the RBs are excitable in wild-type and touchdown mutant larvae. C, Touchdown mutants possess both type I and type generator potential (arrows) at the onset and offset of the stimulus. In these RBs, increasing the amplitude of stimuli eventually triggered action potentials (cyan) at the onset and the offset of the stimulus. In type II RBs, activation by tactile stimuli produced action potentials (cyan) without visible generator potentials (arrowheads) in response to subthreshold stimuli (black).

that labels dead/dying cells (Seiler and Nicolson, 1999). However, no acridine orange staining was observed in the dorsal spinal cord of touchdown mutants.

As a more stringent examination of cell death, the touchdown mutation was crossed into the ssx-mini-ICP:eGFP stable transgenic line of zebrafish, which express eGFP within RBs (Uemura et al., 2005). An analysis of RB gross morphology in this line following immunohistochemical amplification of the eGFP signal revealed a similar number of RBs per hemisegment in touchdown mutants when compared with wild-type siblings (wt, $2.3 \pm 0.6$; vs $t d o, 2.5 \pm 0.3, p=0.8 ; n=6$ fish from two clutches), and no obvious difference in the extent of skin innervation by RBs in touchdown mutants compared with wild-type siblings (Fig. 7). Thus, a loss of sensory neurons does not appear to account for the touch-unresponsive phenotype in touchdown mutants. 
Table 1. Analysis of RB membrane properties from wild-type and touchdown mutant larvae

\begin{tabular}{|c|c|c|c|c|c|c|c|c|c|}
\hline & \multicolumn{3}{|c|}{ Electrical stimulation of cell body } & \multicolumn{3}{|c|}{ Electrical stimulation of peripheral neurite } & \multicolumn{3}{|c|}{$\begin{array}{l}\text { Mechanical stimulation of peripheral } \\
\text { neurite }\end{array}$} \\
\hline & wt & tdo & $p$ & wt & tdo & $p$ & wt & tdo & $p$ \\
\hline Action potential threshold (mV) & $-28.6 \pm 1.5$ & $-29.6 \pm 0.6$ & 0.6 & $-64.4 \pm 2.6$ & $-63.4 \pm 2.5$ & 0.8 & - & - & - \\
\hline Action potential threshold ( $\mu \mathrm{m}$ of displacement) & - & - & - & - & - & - & $24.5 \pm 1.7$ & $22.2 \pm 3.7$ & 0.6 \\
\hline Amplitude of overshoot $(\mathrm{mV})$ & $18.2 \pm 6.7$ & $13.3 \pm 6.9$ & 0.6 & $24.4 \pm 9.6$ & $11.9 \pm 10.3$ & 0.4 & $9.0 \pm 6.0$ & $4.5 \pm 10.9$ & 0.7 \\
\hline Amplitude of undershoot (mV) & $-76.3 \pm 1.3$ & $-69.6 \pm 1.5$ & 0.005 & $-80.0 \pm 1.6$ & $-72.2 \pm 1.0$ & 0.002 & $-72.0 \pm 2.9$ & $-69.9 \pm 1.4$ & 0.6 \\
\hline
\end{tabular}

Each RB was tested with all three types of stimulation so the resting membrane potential data are only shown in the Electrical stimulation of cell body column. Data from Type I and Type II neurons were pooled because no significant difference was observed between them for any of these parameters. $n=10$ for each from several clutches.

\section{Cutaneous mechanoreceptors in touchdown mutants respond to tactile stimuli}

In mammals, TRPM7 has been implicated in mechanosensation (Numata et al., 2007a,b). To test whether TRPM7 plays a similar role in zebrafish sensory neurons, it was first necessary to determine whether there were changes in excitability of RBs in touchdown mutants. We made in vivo whole-cell current-clamp recordings from wild-type and touchdown mutant RBs expressing eGFP in the stable ssx-mini-ICP:eGFP transgenic zebrafish (Fig. 8A). In response to depolarizing current injections to the cell body, RBs from wild-type and touchdown mutants were found to display action potentials that readily overshot $0 \mathrm{mV}$ (Fig. $8 B$ ). An analysis of the resting membrane potential, action potential threshold, and amplitude of over and undershoots failed to uncover differences between wild-type and touchdown mutant RBs (Table 1). This finding is consistent with a previous report detailing the development of ionic currents from an unspecified allele of touchdown (Ribera and Nüsslein-Volhard, 1998). Thus, the loss of TRPM7 in touchdown allele $t d o^{\text {mil }}$ does not affect the excitability of RBs.

Next, the ability of touchdown mutant RBs to respond to electrical and tactile stimulation of the peripheral neurite was explored. We used a recently described in situ preparation (Low et al., 2010b) in which the skin and muscle contralateral to a target $\mathrm{RB}$ were removed, thus providing access to an ipsilateral $\mathrm{RB}$ with intact peripheral neurites (Fig. 8A). A bipolar stimulating probe attached to a piezoelectric actuator was then placed on the skin above eGFP-positive neurites to electrically and mechanically stimulate the same neurite while recording from the cell body. In both wild-type and touchdown mutant RBs, depolarization of the peripheral neurite with a local extracellular electrical stimulus evoked action potentials detectable within the cell body (Fig. 8 B). An examination of the response to peripheral electrical stimulation revealed that wild-type and touchdown mutant RBs exhibited similar action potential thresholds and generated action potentials with similar overshoots (Table 1).

To examine whether tactile stimuli delivered to the peripheral neurite could evoke action potentials that propagated to the cell body, the bipolar stimulating probe was used to mechanically depress the skin above the peripheral neurite with a piezoelectric actuator. Tactile stimulation of peripheral neurites in both wild-type and touchdown mutant RBs evoked action potentials detectable within cell bodies $(n=10 / 10$ each for wt and $t d o$ ) (Fig. 8C). A closer examination revealed that wild-type and touchdown mutants possessed RBs that could be subdivided into two groups, similar to a previous report (Low et al., 2010b). Type I RBs possessed observable generator potentials from which the action potential arose (type I: wt, $n=$ $5 / 10 ; t d o, n=6 / 10)$. In contrast, spikes in type II RBs arose directly from the baseline with no observable generator potential (type II: wt, $n=5 / 10 ; t d o, n=4 / 10)$. The amplitude of mechanical stimuli required to activate both types of RBs was not found to differ signifi- cantly between wild-type and touchdown mutant RBs (Table 1). Thus, wild-type and touchdown mutants possess RBs capable of responding to tactile stimuli in a similar manner. Overall, these results suggest that TRPM7 is required downstream of sensory neuron activation.

\section{Acute elevation of selective divalent cations restores touch-responsiveness in touchdown mutants}

In mammals, TRPM7 has been shown to colocalize with synaptic vesicle proteins at peripheral cholinergic synapses where it potentiates vesicle fusion and transmitter release (Krapivinsky et al., 2006; Brauchi et al., 2008). A similar role for zebrafish TRPM7 at peripheral synapses was ruled out by the ability to restore touchresponsiveness through the selective expression of TRPM7 in sensory neurons. However, one alternate possibility is that TRPM7 plays an analogous role by enhancing transmitter release at the central synapses made by RBs. If true, then treatments that increase the amount of transmitter release from sensory neuron terminals by a different mechanism might ameliorate touchunresponsiveness in touchdown mutants. To explore this idea, we removed the skin above somites 5-10 in touchdown larvae to facilitate rapid equilibration of the spinal cord with the bathing solution, and then elevated the concentration of extracellular $\mathrm{Ca}^{2+}$ from 2 to $5 \mathrm{~mm}$. This high calcium treatment efficiently restored touch-evoked escape behaviors in touchdown mutants (Fig. 9A). In addition to calcium, strontium and, to a lesser extent, barium have been shown capable of facilitating vesicle fusion in fish (Neves et al., 2001). In touchdown mutants, we observed a corresponding ability of solutions containing these divalent cations at $5 \mathrm{~mm}$ to restore touch-evoked behaviors. As a negative control, $5 \mathrm{~mm}$ magnesium, which does not support transmitter release, failed to restore touch-evoked behaviors in touchdown mutants (Fig. 9B). The finding that $\mathrm{Ca}^{2+}, \mathrm{Ba}^{2+}$, and $\mathrm{Sr}^{2+}$ but not $\mathrm{Mg}^{2+}$, rescued touch-evoked behaviors leaves open the interesting, but not yet tested, hypothesis that TRPM7 is released by sensory neurons within the CNS.

\section{Discussion}

The work reported here began with the isolation of a new allele of the zebrafish mutant touchdown that, similar to previously isolated alleles of touchdown, yielded offspring that were hypopigmented and displayed a transient loss of touch-evoked behaviors on the third day of development. We used this new allele of touchdown $\left(t d o^{\text {mil74 }}\right)$ to investigate the in vivo contribution of TRPM7's kinase activity and ion selectivity, and then explored several of the in vivo putative roles for TRPM7.

Domains of TRPM7 required for touch-evoked responses Touchdown alleles $t d o^{\text {mi174 }}$ and $t d o^{b 508}$ arise from mutations that truncate TRPM7 within the six transmembrane spanning region (6TM) before the putative pore and just before the $\mathrm{C}$ terminal 
kinase domain, respectively. In contrast, touchdown allele $t d o^{t z 310 c}$ arises from a missense mutation $\left(\right.$ TRPM7 $\left.7_{\text {L858P }}\right)$ within the second transmembrane domain of the 6TM region and therefore has the potential to form a full-length protein with a mutated ion channel and normal C terminal kinase. Oocyte expression studies revealed a loss of TRPM7 channel activity in all three alleles. The loss of channel function in $t d o^{b 508}$ was not surprising, as previous studies of mammalian TRPM7 have shown that two different truncations beginning near the start of the $\mathrm{C}$ terminal kinase domain lead to a dramatic reduction (Schmitz et al., 2003) or a complete abolition (Matsushita et al., 2005) of current. The latter authors concluded that the effect of the truncations must be due to changes in the structure or targeting of the modified protein rather than due to the loss of the kinase activity, as mammalianTRPM7 with point mutations that abolish kinase activity produce currents with amplitudes similar to wild-type TRPM7 (Nadler et al., 2001; Schmitz et al., 2003; Matsushita et al., 2005).

The finding that the zebrafish TRPM7 ${ }_{\text {D1686A }}$ kinase mutant and TRPM7 ${ }_{\mathrm{E} 1026 \mathrm{Q}}$ ion selectivity mutant both produce large currents in oocytes and rescue touch-responsiveness in touchdown mutants led us to the hypothesis that current flow through TRPM7 is necessary for touch-responsiveness in larval zebrafish. A prediction of this hypothesis is that a nonconducting TRPM7, which was otherwise normal, would be unable to rescue touch-responsiveness in touchdown mutants. Indeed, the TRPM7 $7_{\mathrm{E} 1026 \mathrm{~K}}$ pore mutant produced no detectable currents in oocytes and failed to rescue touch-responsiveness in touchdown mutants. However, the failure to rescue could potentially have been produced indirectly if the mutation made the $\mathrm{TRPM}_{\mathrm{E} 1026 \mathrm{~K}}$ protein abnormal in another way, such as altering protein stability or trafficking to the membrane. One reason for suspecting that the TRPM7 ${ }_{\mathrm{E} 1026 \mathrm{~K}}$ mutation might not alter protein stability or trafficking was the finding that a second mutation at the same site $\left(\mathrm{TRPM} 7_{\mathrm{E} 1026 \mathrm{Q}}\right)$ resulted in a functional protein in oocytes and was capable of rescuing the touchdown phenotype in larval zebrafish. For cells in culture, a standard way to check for mistrafficking is to use Western blotting to detect surface proteins that have been biotinylated on extracellular lysines and then precipitated by strepavidin beads. However, the small number of sensory neurons in larval zebrafish and the inaccessibility of these cells to biotinylating reagents made this approach impractical. Furthermore, even if it could be successfully demonstrated that TRPM7 $7_{\mathrm{E} 1026 \mathrm{~K}}$ is on the surface of sensory neurons, this result would still not unequivocally demonstrate that TRPM7 ion channel activity is necessary for touch-responsiveness in zebrafish, as additional consequences of the E1026K mutation might exist. For instance, the E1026K substitution might prevent TRPM7 from interacting with an unknown binding partner, and it might be this protein-protein interaction that is essential for touchresponsiveness in zebrafish. Regardless, the hypothesis that TRPM7 channel activity is required for proper function of sen- sory neurons in zebrafish remains a simple way to explain all available data.

In conclusion, the rescue of touch-responsiveness in touchdown mutants with a modified TRPM7 with greatly reduced permeability to divalent cations, or one lacking a critical residue necessary for kinase activity, demonstrates that these aspects of TRPM7 are dispensable for this behavior.

\section{The role of TRPM7 in the escape circuit}

The finding that selective expression of wild-type TRPM7 in touchdown mutant sensory neurons was sufficient to rescue touch-evoked responses, and that normal fictive swimming can be evoked by NMDA in touchdown mutants, suggest that the interneurons postsynaptic to sensory neurons are normal in mutants. We therefore performed experiments to explore why TRPM7 was required in sensory neurons. In vivo electrophysiological recordings indicated that important membrane properties of RBs were unchanged in mutant larvae and that TRPM7 was not required for mechanical activation of RBs. However, the observation that tactile stimulation in touchdown mutants failed to evoke spikes in Mauthner cells, coupled with the reported participation of mammalian TRPM7 in neurotransmitter release at peripheral synapses (Krapivinsky et al., 2006; Brauchi et al., 2008), led us to consider the possibility that communication between sensory neurons and second-order neurons might be altered in TRPM7 mutants.

At this time, our findings do not explicitly test a role for TRPM7 within synaptic vesicles in sensory neurons similar to that proposed for mammalian TRPM7 at peripheral synapses, so this is simply an appealing hypothesis. However, if the role of 
TRPM7 is to facilitate transmitter release at sensory neuron to interneuron synapses, then what could account for the early and late periods of touch-responsiveness in touchdown mutants? One possible explanation is that the resistance of zebrafish neurons decreases during development (Saint-Amant and Drapeau, 2001), likely as a consequence of an increase in cell size, resulting in an increase in the amount of synaptic drive required to achieve threshold. If the role of TRPM7 in zebrafish sensory neurons is to facilitate transmitter release, then a small amount of transmitter might still be released in the absence of TRPM7, an amount sufficient to drive critical interneurons past threshold when neurons are small. This small amount of transmitter would become insufficient to drive interneurons past threshold as the neurons grow. A similar model involving a decrease in neuron resistance has been proposed to explain the progressive inability of gap junction-mediated periodic depolarizations within the embryonic zebrafish central pattern generator to drive motor neurons to threshold during the extinction phase of spontaneous contractions (Saint-Amant and Drapeau, 2000). A second possible explanation is that maternally derived mRNA produces sufficient wild-type TRPM7 protein to support early touch-evoked responses. Because the TRPM7 MO failed to suppress the early touch-evoked responses, this seems unlikely. MO experiments also appeared to rule out a required role for the closely related protein TRPM6 in eliciting early touch-evoked behaviors. Because MO knockdowns may not be effective at later stages of development, it is still unclear whether TRPM6 could account for the tactile responsiveness observed in mutants at late stages. Of course, there remains the possibility that other molecular classes of channels are developmentally regulated in a pattern that allows zebrafish to produce touch-evoked responses without TRPM7 at certain stages of development.

In summary, we have shown that TRPM7 is transiently required within sensory neurons for the activation of touch-evoked behaviors, and an attractive hypothesis to explain this observation is that TRPM7 potentiates transmitter release at central synapses between sensory neurons and interneurons, similar to the role of mammalian TRPM7 at peripheral synapses. To determine whether TRPM7 contributes to transmitter release at central synapses will require electrophysiological recordings from not yet identified interneurons directly postsynaptic to RBs.

\section{References}

Arduini BL, Henion PD (2004) Melanophore sublineage-specific requirement for zebrafish touchtone during neural crest development. Mech Dev 121:1353-1364.

Bernhardt RR, Chitnis AB, Lindamer L, Kuwada JY (1990) Identification of spinal neurons in the embryonic and larval zebrafish. J Comp Neurol 302:603-616.

Brauchi S, Krapivinsky G, Krapivinsky L, Clapham DE (2008) TRPM7 facilitates cholinergic vesicle fusion with the plasma membrane. Proc Natl Acad Sci U S A 105:8304-8308.

Buss RR, Drapeau P (2000) Physiological properties of zebrafish embryonic red and white muscle fibers during early development. J Neurophysiol 84:1545-1557.

Chubanov V, Waldegger S, Mederos y Schnitzler M, Vitzthum H, Sassen MC, Seyberth HW, Konrad M, Gudermann T (2004) Disruption of TRPM6/ TRPM7 complex formation by a mutation in the TRPM6 gene causes hypomagnesemia with secondary hypocalcemia. Proc Natl Acad Sci U S A 101:2894-2899.

Clark K, Langeslag M, van Leeuwen B, Ran L, Ryazanov AG, Figdor CG, Moolenaar WH, Jalink K, van Leeuwen FN (2006) TRPM7, a novel regulator of actomyosin contractility and cell adhesion. EMBO J 25:290-301.

Clark K, Middelbeek J, Lasonder E, Dulyaninova NG, Morrice NA, Ryazanov AG, Bresnick AR, Figdor CG, van Leeuwen FN (2008) TRPM7 regulates myosin IIA filament stability and protein localization by heavy chain phosphorylation. J Mol Biol 378:790-803.
Cui WW, Saint-Amant L, Kuwada JY (2004) shocked Gene is required for the function of a premotor network in the zebrafish CNS. J Neurophysiol 92:2898-2908.

Dorovkov MV, Ryazanov AG (2004) Phosphorylation of annexin I by TRPM7 channel-kinase. J Biol Chem 279:50643-50646.

Drapeau P, Ali DW, Buss RR, Saint-Amant L (1999) In vivo recording from identifiable neurons of the locomotor network in the developing zebrafish. J Neurosci Methods 88:1-13.

Eaton RC, Nissanov J, Wieland CM (1984) Differential activation of Mauthner and non-Mauthner startle circuits in the zebrafish: implications for functional substitution. J Comp Physiol A Neuroethol Sens Neural Behav Physiol 155:813-820.

Elizondo MR, Arduini BL, Paulsen J, MacDonald EL, Sabel JL, Henion PD, Cornell RA, Parichy DM (2005) Defective skeletogenesis with kidney stone formation in dwarf zebrafish mutant for trpm7. Curr Biol 15:667-671.

Gahtan E, Sankrithi N, Campos JB, O’Malley DM (2002) Evidence for a widespread brain stem escape network in larval zebrafish. J Neurophysiol $87: 608-614$.

Granato M, van Eeden FJ, Schach U, Trowe T, Brand M, Furutani-Seiki M, Haffter P, Hammerschmidt M, Heisenberg CP, Jiang YJ, Kane DA, Kelsh RN, Mullins MC, Odenthal J, Nüsslein-Volhard C (1996) Genes controlling and mediating locomotion behavior of the zebrafish embryo and larva. Development 123:399-413.

Haffter P, Nüsslein-Volhard C (1996) Large scale genetics in a small vertebrate, the zebrafish. Int J Dev Biol 40:221-227.

Jin J, Desai BN, Navarro B, Donovan A, Andrews NC, Clapham DE (2008) Deletion of Trpm7 disrupts embryonic development and thymopoiesis without altering $\mathrm{Mg} 2+$ homeostasis. Science 322:756-760.

Jontes JD, Buchanan J, Smith SJ (2000) Growth cone and dendrite dynamics in zebrafish embryos: early events in synaptogenesis imaged in vivo. Nat Neurosci 3:231-237.

Kim BJ, Lim HH, Yang DK, Jun JY, Chang IY, Park CS, So I, Stanfield PR, Kim KW (2005) Melastatin-type transient receptor potential channel 7 is required for intestinal pacemaking activity. Gastroenterology 129:15041517.

Kimmel CB, Ballard WW, Kimmel SR, Ullmann B, Schilling TF (1995) Stages of embryonic development of the zebrafish. Dev Dyn 203:253-310.

Korn H, Faber DS (2005) The Mauthner cell half a century later: a neurobiological model for decision-making? Neuron 47:13-28.

Krapivinsky G, Mochida S, Krapivinsky L, Cibulsky SM, Clapham DE (2006) The TRPM7 ion channel functions in cholinergic synaptic vesicles and affects transmitter release. Neuron 52:485-496.

Li M, Du J, Jiang J, Ratzan W, Su LT, Runnels LW, Yue L (2007) Molecular determinants of $\mathrm{Mg} 2+$ and $\mathrm{Ca} 2+$ permeability and $\mathrm{pH}$ sensitivity in TRPM6 and TRPM7. J Biol Chem 282:25817-25830.

Low SE, Zhou W, Choong I, Saint-Amant L, Sprague SM, Hirata H, Cui WW, Hume RI, Kuwada JY (2010a) Na(v)1.6a is required for normal activation of motor circuits normally excited by tactile stimulation. Dev Neurobiol 70:508-522.

Low SE, Ryan J, Sprague SM, Hirata H, Cui WW, Zhou W, Hume RI, Kuwada JY, Saint-Amant L (2010b) touche Is required for touch-evoked generator potentials within vertebrate sensory neurons. J Neurosci 30:9359-9367.

Matsushita M, Kozak JA, Shimizu Y, McLachlin DT, Yamaguchi H, Wei FY, Tomizawa K, Matsui H, Chait BT, Cahalan MD, Nairn AC (2005) Channel function is dissociated from the intrinsic kinase activity and autophosphorylation of TRPM7/ChaK1. J Biol Chem 280:20793-20803.

Mongeon R, Gleason MR, Masino MA, Fetcho JR, Mandel G, Brehm P, Dallman JE (2008) Synaptic homeostasis in a zebrafish glial glycine transporter mutant. J Neurophysiol 100:1716-1723.

Montell C (2005) The TRP superfamily of cation channels. Sci STKE 2005:re3.

Nadler MJ, Hermosura MC, Inabe K, Perraud AL, Zhu Q, Stokes AJ, Kurosaki T, Kinet JP, Penner R, Scharenberg AM, Fleig A (2001) LTRPC7 is a Mg.ATP-regulated divalent cation channel required for cell viability. Nature 411:590-595.

Neves G, Neef A, Lagnado L (2001) The actions of barium and strontium on exocytosis and endocytosis in the synaptic terminal of goldfish bipolar cells. J Physiol 535:809-824. 
Numata T, Shimizu T, Okada Y (2007a) Direct mechano-stress sensitivity of TRPM7 channel. Cell Physiol Biochem 19:1-8.

Numata T, Shimizu T, Okada Y (2007b) TRPM7 is a stretch- and swellingactivated cation channel involved in volume regulation in human epithelial cells. Am J Physiol Cell Physiol 292:C460 - 467.

Prugh JI, Kimmel CB, Metcalfe WK (1982) Noninvasive recording of the Mauthner neurone action potential in larval zebrafish. J Exp Biol 101:83-92.

Ribera AB, Nüsslein-Volhard C (1998) Zebrafish touch-insensitive mutants reveal an essential role for the developmental regulation of sodium current. J Neurosci 18:9181-9191.

Runnels LW, Yue L, Clapham DE (2001) TRP-PLIK, a bifunctional protein with kinase and ion channel activities. Science 291:1043-1047.

Saint-Amant L, Drapeau P (1998) Time course of the development of motor behaviors in the zebrafish embryo. J Neurobiol 37:622-632.

Saint-Amant L, Drapeau P (2000) Motoneuron activity patterns related to the earliest behavior of the zebrafish embryo. J Neurosci 20:3964-3972.

Saint-Amant L, Drapeau P (2001) Synchronization of an embryonic net- work of identified spinal interneurons solely by electrical coupling. Neuron 31:1035-1046.

Schmitz C, Perraud AL, Johnson CO, Inabe K, Smith MK, Penner R, Kurosaki T, Fleig A, Scharenberg AM (2003) Regulation of vertebrate cellular Mg2+ homeostasis by TRPM7. Cell 114:191-200.

Seiler C, Nicolson T (1999) Defective calmodulin-dependent rapid apical endocytosis in zebrafish sensory hair cell mutants. J Neurobiol 41:424-434.

Uemura O, Okada Y, Ando H, Guedj M, Higashijima S, Shimazaki T, Chino N, Okano H, Okamoto H (2005) Comparative functional genomics revealed conservation and diversification of three enhancers of the isl1 gene for motor and sensory neuron-specific expression. Dev Biol 278:587-606.

Westerfield M (2000) The zebrafish book: a guide for the laboratory use of zebrafish (Danio rerio), 4th Edition. Eugene: University of Oregon.

Zottoli SJ, Faber DS (1979) Properties and distribution of anterior VIIIth nerve excitatory inputs to the goldfish Mauthner cell. Brain Res 174:319323. 